\title{
Androgen suppression duration and zoledronic acid: under the RADAR
}

E ach year, more than 5,000 men in Australia and New Zealand are diagnosed with locally advanced prostate cancer. Unfortunately, in at least $25 \%$ of cases, the cancer has metastasized to the bone and lymph glands, and is undetectable by current imaging methods. As a result, these men cannot undergo active surveillance and instead require treatment for prolonged periods. Although longterm androgen suppression following radiotherapy has been shown to be more effective than short-term androgen suppression (STAS), longerterm treatment is also associated with greater morbidities, such as osteopenia and fractures. The Trans-Tasman Radiation Oncology Group (TROG) 96.01 trial determined the value of 6 months of STAS. In the USA and Europe, androgen suppression lasting 28-36 months was frequently used after other trials showed that longer duration could reduce prostate cancer mortality. However, long-term and sometimes permanent side effects included sexual dysfunction, hot flushes, breast enlargement and pain, shrinkage of the genitalia, anaemia, blood clots, fatigue, cognitive dysfunction, depression and reductions in bone mineral density resulting in fractures.

"With these long-term testosterone suppression side effects in mind, we designed the TROG 03.04 RADAR trial to determine whether better outcomes could be achieved with intermediateterm androgen suppression (ITAS) and radiotherapy without all of these side effects," explains James Denham, lead investigator of the RADAR study. Since the bisphosphonate zoledronic acid has anticancer properties in men with castrationresistant prostate cancer and bone metastases, the RADAR trial was initiated to compare STAS with ITAS with or without zoledronic acid. This randomized, open-label, phase III trial with a

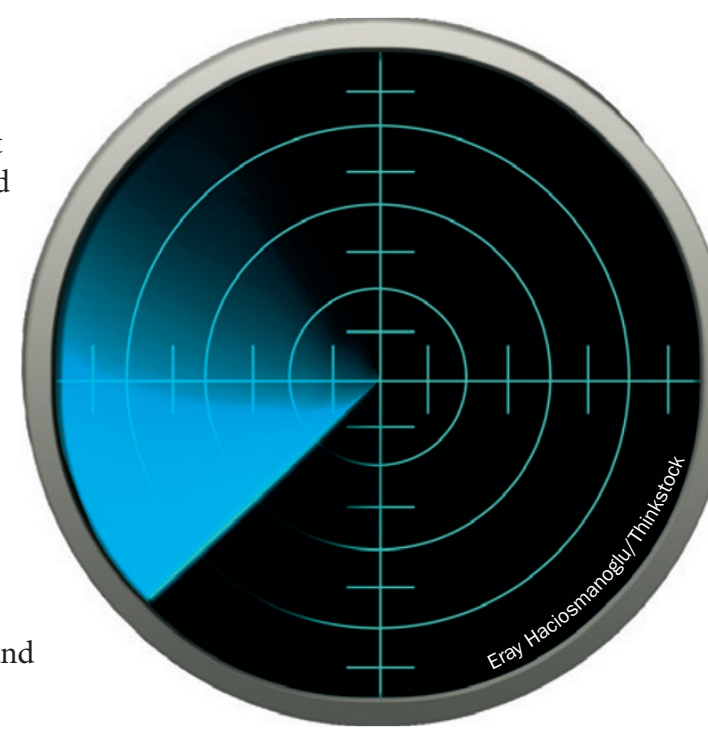

cautiously as a preventative measure against the development of metastases," explains Denham.

As these findings were based on secondary end point data from the trial, the authors point out that caution is needed when interpreting such data. External validation of the interaction between zoledronic acid and Gleason score is, therefore, needed. For men with Gleason score 8-10, 18 months of leuprorelin and zoledronic acid commencing 5 months before radiotherapy reduces metastatic spread by more than $40 \%$. By contrast, in men with Gleason score $\leq 7$ tumours, 18 months of leuprorelin might be the best option.

"We want to confirm the interaction between Gleason score and the use of zoledronic acid seen in the RADAR trial using a bone model because it could be very important from a biological perspective as well as the immediate clinical perspective," adds Denham. His team "is hoping to collaborate with other trialists evaluating the role of zoledronic acid in preventing prostate cancer bone metastasis, by conducting a meta-analysis that has exploration of the RADAR trial interaction as one of its goals."

Although further follow-up data from international trials are needed to establish the value of ITAS with or without zoledronic acid, the RADAR trial highlights that zoledronic acid benefits men at highest risk, but should be spared in men with Gleason score $\leq 7$ tumours.

\section{Lisa Hutchinson}

This article was originally published in Nat. Rev. Clin. Oncol. (doi:10.1038/nrclinonc.2014.150) zoledronic acid did not help. Of concern, those who received zoledronic acid and 6 months androgen suppression experienced a small but significant increase in bone metastases of approximately $1 \%$ per year. This effect was more pronounced in men with Gleason score $\leq 7$ tumours. Zoledronic acid must therefore be used
Original article Denham, J. W. et al. Short-term androgen suppression and radiotherapy versus intermediate-term androgen suppression and radiotherapy, with or without zoledronic acid, in men with locally advanced prostate cancer (TROG 03.04 RADAR): an open-label, randomised, phase 3 factorial trial. Lancet Oncol. doi:10.1016/ S1470-2045(14)70328-6 